\title{
Estrategia de análisis de obra interactiva: el laboratorio alternativo como herramienta*
}

\author{
José Antonio Dorado Zúñiga** \\ Javier M. Reyes Vera*** \\ Ana García López $z^{* * *}$ \\ Recibido: 2020-07-02 • Enviado a pares: 2020-07-28 \\ Aprobado por pares: 2020-08-29 • Aceptado: 2020-09-08 \\ https://doi.org/10.22395/angr.v19n38a5
}

\section{Resumen}

La instalación interactiva constituye actualmente una alternativa importante para difundir obras en museos y convocar nuevos públicos, pero una de sus limitaciones es diagnosticar a tiempo problemas potenciales que puedan darse en el proceso de comunicación interactivo. Frente a esta hipótesis de partida, se planteó como objetivo la creación de un laboratorio audiovisual pedagógico capaz de evaluar y diagnosticar posibles dificultades en dicho proceso comunicativo y ayudar a establecer las estrategias para adelantar propuestas alternativas y mejorar la interacción entra la obra y el público. En consecuencia, se adecuó un laboratorio de ensayo en un estudio de televisión donde se aplicó la prueba emot-brain: Método de evaluación de experiencia de usuario a la instalación Uno se muere y sigue aprendiendo a pintar con un grupo focal de estudiantes y profesores de la universidad del Valle. El ensayo arrojó indicadores tímicos que se registraron a través del dispositivo Emotiv-epoc y relaciones cronémicas y proxémicas que se sistematizaron a través de la observación participante, cámaras infrarrojas y de alta sensibilidad y entrevistas. Las evaluaciones arrojaron evidencias de las fortalezas y limitaciones en la praxis de las obras expuestas y permitieron advertir, comprender y enriquecer las relaciones interactivas. Con estos insumos se ajustaron los correctivos y se difundió la obra. La experiencia constituye un recurso de apoyo para orientar a estudiantes y creadores frente a la incertidumbre sobre la funcionalidad de sus obras con pruebas para apoyar procesos creativos. De esta manera, se revelan aspectos que los creadores no advierten en la concepción de las obras.

Palabras clave: artes; exposición; ensayo; instalación cultural; pintura; interacción hombre-máquina.

\footnotetext{
Este artículo es resultado de investigación y proyecto de creación de una obra interactiva museística en la Escuela de Comunicación Social de la Universidad del Valle, que hace parte de los trabajos conducentes a la realización de la tesis doctoral en la Universidad de Granada (España) y el proyecto de investigación denominado UX-Brain: evaluación de la experiencia de usuario del uso de herramientas cotidianas basado en sus estados cerebrales (Rodríguez, 2013; Velásquez, 2012) desarrollada en el Departamento de Diseño de la Universidad del Valle, Cali, Colombia.

. Candidato a doctor en Historia y Artes, Universidad de Granada, Granada, España. Magíster en Literatura Colombiana y Latinoamericana, Universidad del Valle, Cali, Colombia. Director de cine, especialista en Prácticas Audiovisuales, docente e investigador de la Escuela de Comunicación Social, Facultad de Artes Integradas, Universidad del Valle, Cali, Colombia. Correo electrónico: jose.dorado@ correounivalle.edu.co. Orcid: https://orcid.org/0000-0001-7372-9378

... Doctor en Ciencias de la Computación, Universidad del Valle, Cali, Colombia. Magíster en Ingeniería, Universidad del Valle, Cali, Colombia. Especialista en Diseño de Ambientes Virtuales de Aprendizaje, Uniminuto, Bogotá, Colombia. Diseñador industrial, Universidad Industrial de Santander, Bucaramanga, Colombia. Investigador grupo Camaleón y docente, Universidad del Valle, Cali, Colombia. Correo electrónico: javier.reyes@correounivalle.edu.co. Orcid: https://orcid.org/0000-0001-8486-388X

.... Doctora en Historia y Artes, Universidad de Granada, Granada, España. Licenciada en Bellas Artes, Universidad de Granada, Granada, España. Docente e investigadora en el Departamento de Dibujo, Universidad de Granada, Granada, España. Correo electrónico: agarcial@ ugr.es. Orcid: https://orcid.org/0000-0003-3274-6651
} 


\title{
Interactive Work Analysis Strategy: the Alternative Laboratory as a Tool
}

\begin{abstract}
The interactive installation is currently an important alternative for disseminating works in museums and calling on new audiences, but one of its limitations is the early diagnosis of potential problems that may occur in the interactive communication process. Faced with this initial hypothesis, the aim of this work was to create an educational audiovisual laboratory capable of evaluating and diagnosing possible difficulties in this communicative process and help to establish strategies to advance alternative proposals and improve the interaction between the works at the museum and the public. As a result, a test laboratory was adapted in a television studio where the emot-brain test was applied: a method for evaluating user experience to the installation -Uno se muere y sigue aprendiendo a pintar- (Even dead one keeps learning how to paint, in English) with a focus group of students and professors from Universidad del Valle. The test yielded thymic indicators that were recorded through the Emotiv-epoc ${ }^{\circledR}$ device and chronemic and proxemic relationships that were systematized through participant observation, infrared and high sensitivity cameras and interviews. The evaluations provided evidence of the practical strengths and limitations of the works exhibited and allowed the interactive relationships to be noticed, understood and enriched. With these inputs, the corrections were adjusted, and the work was disseminated. The experience constitutes a support resource to guide students and creators in the face of uncertainty about the functionality of their works with evidence to support creative processes, revealing aspects that creators do not notice in the conception of the works.
\end{abstract}

Keywords: arts; exhibition; test; cultural installation; painting; human computer interaction. 


\section{Introducción}

La experiencia surgió a partir de la necesidad de verificar, orientar y refinar decisiones sobre la instalación interactiva Uno se muere y sigue aprendiendo a pintar, inspirada en la obra del artista plástico Augusto Rivera (1922-1982), uno de los pintores indigenistas más importantes de la plástica nacional. La creación conceptual y estética de la instalación surgió de indagar la estilística de las pantallas expandidas, las alternativas de dispositivos interactivos y su correlación con el régimen escópico del artista (Dorado, 2016).

De esta manera, frente a la incertidumbre de los resultados, se propuso unificar esfuerzos para emplear el análisis como una herramienta pedagógica e investigativa fusionada en lo que denominamos laboratorio de creación artística. La propuesta está inspirada en el espíritu formativo y experimental de Lev Kulechov (Mariniello, 1992) y la influencia de las vanguardias en los nuevos medios (Manovich, 2005). El propósito de esta propuesta es hacer una reinterpretación del desplazamiento de la observación participante al laboratorio para hacer evidente el objeto de estudio (Latour, 1983) y apropiar elementos de los laboratorios contemporáneos interesados en indagaciones de interacciones hápticas o interdisciplinares en el ámbito artístico y educativo (Sedeño, 2015, p. 184).

Así, el salón de clase se extrapoló a un laboratorio pedagógico que permitió poner a prueba los dispositivos interactivos, además de aplicar una pruebas y realizar de manera complementaria un ejercicio pragmático de observación etnográfica (Restrepo, 2016). La propuesta creó un escenario donde el artista, el estudiante, el docente y el público compartieron una experiencia formativa y creativa. Se adaptó el principio pedagógico de educar/crear en comunidad con la premisa de que "ahora, ya nadie educa a nadie, así como tampoco nadie se educa a sí mismo, los hombres se educan en comunión, y el mundo es el mediador" (Freire, 2011, p. 61).

Como laboratorio pedagógico, se adaptó el estudio de televisión del canal universitario - con un área de ciento veinte metros cuadrados- que se ajustó a las necesidades del montaje espacial y tecnológico. La aplicación de la herramienta UX-Brain, método de evaluación de la experiencia en la interacción con elementos de uso cotidiano a partir de los estados emocionales, permitió hacer seguimiento y valoración de comportamientos tímicos ${ }^{1} \mathrm{o}$

Por oposición a la dimensión cognitiva que está relacionada con el conocimiento, la dimensión tímica está relacionada con la emoción. En el caso de la presente investigación nos permite establecer valores de aceptación, rechazo o indiferencia. El Centro Virtual Cervantes (2008) lo refiere como: estado anímico básico preponderante. 
proxémicos ${ }^{2}$ además de inferir relaciones entre usuario-obra —intangibles o manipulables- (Roger, 2003; Tao, 2005). De igual manera, se empleó un sistema de diademas $\mathrm{BCI}$ que se ofrecieron aleatoriamente al público para portarla en los recorridos. Estos dispositivos arrojaron índices a través de un algoritmo diseñado para el laboratorio interactivo que permitió capturar diferentes estados del cerebro al ser expuesto a las diferentes obras. Este recurso detectó el estado de atención que determina la concentración, y el estado de meditación que determina la confianza o el nivel de impacto que se registró. El algoritmo, a su vez, permitió que estos dos estados emitieran variables independientes, el cual toleró que se arrojaran índices que permitieran inferir y evaluar reacciones tímicas del público. Estas informaciones estuvieron predeterminadas por variables alfa, hi-alfa, beta y teta, etc. que generaron valores complementarios sobre estados emocionales de la actividad cerebral.

Como los procesos de interactividad estaban mediados por proyecciones, paisajes sonoros y nuevas tecnologías, se buscó vincular una relación integral entre techne (saber hacer) y poiein (hacer) asumiendo la interpretación que les otorga David Bordwell (1999):

Techne es el término aristotélico para indicar unidad de teoría y práctica dentro de una especialización. [...] El término (procedente de poiesis, es decir, "actividad", "realización", o también "hacer") hace hincapié en entender la obra de arte como el producto de una actividad creativa con vistas a un uso o efecto (pp. 56-57).

Esto quiere decir que, como procesos creativos y reflexivos, se buscaron puentes dialógicos teórico-prácticos entre la obra experimental de Augusto Rivera y el contexto experimental contemporánea del laboratorio.

En consecuencia, el artículo tiene la siguiente estructura: metodología, descripción de la instalación y orientación del recorrido en el laboratorio; conceptos de evaluación de la interactividad; guion de tareas; modelo conceptual en la instalación; plan de evaluación; análisis general de la aplicación de la evaluación; arquitectura del algoritmo UX-Brain; aplicación del método UX-Brain; reelaboración de la propuesta, socialización de resultados; difusión de resultados y conclusiones.

El término proviene del latín proximus que quiere decir más cercano. Se asume como "el estudio del uso y percepción del espacio social y personal" (Knapp, 2009, p. 25). Se emplea como una herramienta de la comunicación no verbal que estudia las maneras como las personas estructuran su relación con los objetos y el espacio. En este caso, como el proceso de interacción es un evento museístico, se evalúa la proximidad, el distanciamiento, la presencia o ausencia del contacto físico haciendo la claridad de que estas relaciones obedecen también a un contexto cultural. Esta categoría es complementaria de la kinesis que estudia los gestos a partir de las expresiones faciales. 


\section{Metodología}

Se integraron metodologías cuantitativas y cualitativas de investigación yuxtaponiendo herramientas etnográficas que apelan al método de observación participante y entrevistas focales (Lin, 2013; Penny, 2011; Guber, 2001). Estas fueron implementadas con el apoyo de estudiantes y docentes que participaron en la investigación. Para afianzar el proceso de reflexividad, en la observación asistida se emplearon dos cámaras infrarrojas y tres pequeñas cámaras luminosas. La penumbra se convirtió en aliado porque ayudó a ocultar o mimetizar los equipos y las personas que los manipulaban. De esta manera, se revelaron reacciones del público frente a la interacción, apropiación espacial y los registros cronométricos demandados en los recorridos por las obras.

En cuanto a la metodología cualitativa, se apeló al uso de tecnologías para la medición y valoración del comportamiento de las personas en relación con los objetos de uso cotidiano y la medición in situ del grado de atención a partir de instrumentos tecnológicos como el Emotive-epoc ${ }^{\circledast}$, pero también el seguimiento de cronometrías de todo el proceso para verificar los impactos.

\section{Descripción de la instalación y orientación del recorrido en el laboratorio}

Se diseñó un recorrido a partir de los cuatro elementos expuestos en un ambiente en un ambiente de penumbra y niebla, iluminados por las proyecciones de la exposición. No obstante lo anterior, la propuesta inducía a los usuarios a improvisar rutas (ver figura 1).

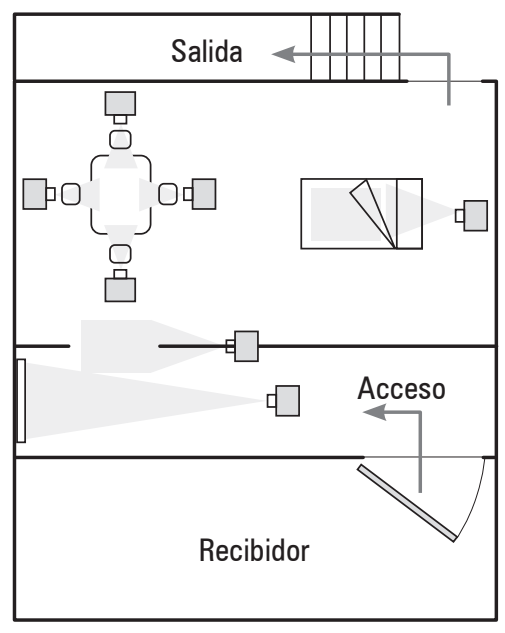

Figura 1. Disposición de la instalación Fuente: elaboración propia. 
El primer elemento tenía la función de introducir al espectador a partir de la proyección de un videoarte, acompañado por la nota biográfica, sinopsis de la instalación interactiva y ficha técnica. En el plano sonoro se colocó una de las grabaciones de la voz de Augusto Rivera en la que problematiza, ebrio y lúcido, su lucha con el arte.

El segundo elemento expuesto se titulaba Senderos de Rivera. Se trataba de un Arduino ${ }^{\circledR}$ que, al detectar la presencia del público, proyectaba una animación de cuatro líneas que progresivamente dibujaban en el piso sobre un humo discreto. Esta proyección aludía a una metáfora de pinceladas continuas que cumplían la función de orientar potenciales rutas del espectador en la instalación. Las líneas eran de colores rojo, blanco, amarillo y azul. Estos tonos fueron seleccionados a partir de la paleta de colores que Rivera empleó en la última etapa de su vida.

El tercer elemento expuesto se denominó Alumbramientos del artista. Cuando el espectador se acercaba en la penumbra, se activaba el sonido de los acordes del Réquiem III de Verdi. Al mismo tiempo, se proyectaban obras de Augusto Rivera sobre una cama cubierta con un lienzo. La cama para el artista no era un lugar de descanso, sino un territorio de batalla, de búsqueda creativa. En la cama paría sus ideas, idealizaba sus sueños y enfrentaba sus pesadillas. La instalación resignifica y transforma un mueble convencional en un espacio simbólico en el que se proyectaban obras de diversas épocas y técnicas ${ }^{3}$ (el trabajo pictórico de Rivera fue producido en un período muy competido con pares como Obregón, Grau o Botero).

El cuarto elemento de exposición era La cena repentista que tenía por mantel un marco forrado en lienzo y cuatro asientos. Este era otro elemento doméstico con varios significados implícitos. En primera instancia, significaba un homenaje al espíritu repentista de Rivera, quien recurría a elementos que tuviera a mano para expresar sus conflictos o estados de ánimo. Pintaba sobre tela, cartón, manteles, cáscaras de huevo (en su infancia). Incluso, hay varios retratos pintados sobre servilletas de tela de restaurantes, pero también hay retratos donde usaba cartón en lugar de lienzo; en lugar de pincel, apelaba a sus propios dedos; y en lugar de óleo o pintura recurría al betún y griffin.

Pero esta pieza de la instalación reclamaba la participación del público: si alguien se acercaba a la mesa, se proyectaba una imagen sobre un lienzo dispuesto de manera ambigua como si fuera un mantel; si un espectador se sentaba en una silla, se proyectaba una imagen sobre el correspondiente plato que estaba en la

Algunas de las obras que se proyectaban en el lienzo-cama le permitieron a Rivera el reconocimiento de la crítica Marta Traba y la curaduría internacional de su obra en museos como el Moma en New York. 
mesa y sobre las servilletas de tela; si se sentaban más espectadores, aparecían más proyecciones sobre cada puesto. Las obras elegidas para las proyecciones correspondían principalmente al período de los años setenta hasta 1982, año en que muere. Encima de la mesa, se colocaron cuatro audífonos, donde se escuchaban grabaciones de la voz del artista exponiendo sus ideas en torno al arte y la creación.

Finalmente, la evacuación de la exposición estaba ubicada hacia el fondo derecho del estudio al subir unas gradas que permitían tener una visión panorámica de los elementos de exposición en conjunto. Luego, las personas eran recibidas en el cuarto de edición del estudio por el personal del equipo de investigación para la realización de una entrevista relacionada con la percepción general de la obra.

\section{Evaluación de la interactividad}

Para la evaluación fue necesario tener presentes dos conceptos (Mahrin, 2009):

- El guion de tareas: representa las acciones (en el contexto del presente artículo se traducen en subactividades) que el público realizó en la instalación (Heimonen et al., 2008). Como se explicó en el apartado anterior, la instalación contó con cuatro elementos dispuestos de forma que el público pudiera apreciar elementos conceptuales y constitutivos de la obra de Augusto Rivera.

- El modelo conceptual: se concibe como la manera adecuada en la que el público debería interactuar con los elementos de la instalación, el recorrido y la percepción general al salir. Este modelo conceptual es la base para la creación, pues ofrece un orden secuencial sinérgico de todos los elementos dispuestos en la instalación. Esto quiere decir que no eran accidentales, sino que estaban allí por una razón definida y concreta.

\section{Guion de tareas y modelo conceptual en la instalación:}

El ambiente de penumbra y niebla que primó en toda la obra generaba contrastes que permitían al público discernir dónde se encontraban los elementos de la instalación y se establecían interpretaciones. La tarea del público era, entonces, interactuar con el espacio y percibir las indicaciones que se ofrecían por diferentes medios (audio, imagen, iluminación) en la instalación interactiva.

El público realizó las siguientes subactividades en la instalación:

I. Ingreso: mediado por un control que permite un flujo específico de personas. Se buscaron condiciones óptimas para observar y comprender con mesura la exposición de los objetos. 
II. Observación de un video de presentación (introducción) dispuesto en una pantalla LCD: este video buscó contextualizar al artista Augusto Rivera.

III. Recorrido guiado por luces proyectadas en el piso (propuestas como Senderos de Rivera) o por el flujo de los demás asistentes: se buscó que las personas interactuaran con las obras de manera libre, sin restricción ni orden. El discurso de toda la instalación podía ser armado al final del recorrido, luego de conocer todos los elementos.

IV. Identificación de la cama presente (Alumbramientos del artista): no había ningún elemento que sugiera acostarse, pues se buscó la contemplación. Sin embargo, los asistentes podían hacerlo si querían.

V. Identificación de la relación entre la cama, el sonido y las proyecciones: acción de orden cognitivo.

VI. Identificación del comedor (la cena repentista): la mesa recibía proyecciones en los platos que se activaban una vez el asistente se sentaba en las respectivas sillas.

VII. Sentarse en las sillas de la mesa y usar audífonos dispuestos: no había ningún elemento que indicara que las personas podían sentarse (al hacerlo, los sensores de las sillas activan proyección y audio). Se dejaba a la intuición del asistente la probabilidad de ignorar parcial o talmente la interacción del video y audio.

VIII. Observación de las proyecciones en los platos al tiempo que se escucha el audio: como se indicó, las proyecciones y el audio estaban sincronizados; una interacción ideal supondría observar las proyecciones al tiempo que se escuchan los audios.

IX. Buscar la salida de la instalación: en la penumbra, esta subactividad se dificultó, pues encontrar la salida implicaba pasar por un cuarto de equipos y usar una puerta diferente a la de acceso. Se tenía la sensación de estar saliendo por la puerta de atrás. En este lugar, los asistentes comentaron sus impresiones sobre la instalación a un grupo de auxiliares de investigación.

\section{Plan de evaluación}

A partir de las alternativas de evaluación y del carácter de la obra, se diseñó esta herramienta básica (tabla 1) que permite considerar todas las variables relevantes en el proceso de evaluación de la instalación (Navarro et al., 2017; Madan y Kumar, 2012). 
Tabla 1. Análisis de interacción

\begin{tabular}{|c|c|}
\hline Objetivo & Analizar la interacción de la instalación de Augusto Rivera. \\
\hline Reseña & $\begin{array}{l}\text { El asistente debe ingresar a la instalación y dejarse llevar por todos los elementos } \\
\text { dispuestos dentro de esta. No se le da más información que la del proyecto de } \\
\text { investigación-creación aprobado por la Vicerrectoría de Investigaciones de la } \\
\text { Universidad del Valle, que tiene que ver con el artista Augusto Rivera. }\end{array}$ \\
\hline Población & $\begin{array}{l}\text { Público general interesado en asistir, estudiantes universitarios, profesionales, } \\
\text { allegados o no al artista. Sus edades oscilan entre veintidós y ochenta años, de } \\
\text { ambos sexos, con diversos niveles de escolaridad. }\end{array}$ \\
\hline $\begin{array}{l}\text { Proceso de } \\
\text { evaluación }\end{array}$ & $\begin{array}{l}\text { Mientras el público interactúa con la instalación, observará todos sus elementos. } \\
\text { Al final de la sesión se harán una entrevista abierta y un registro en video para } \\
\text { su posterior análisis. }\end{array}$ \\
\hline $\begin{array}{l}\text { Objetivo de la } \\
\text { instalación }\end{array}$ & $\begin{array}{l}\text { Proveer una experiencia granular frente a la obra de Augusto Rivera donde se tenga } \\
\text { más información, dependiendo de lo deseado, a partir del uso de herramientas } \\
\text { tecnológicas (sensores, proyectores, pantallas LCD, audio, olores, humo, etc.) que } \\
\text { potencian la experiencia del público. }\end{array}$ \\
\hline $\begin{array}{l}\text { Consentimiento } \\
\text { informado }\end{array}$ & $\begin{array}{l}\text { Se solicitará verbalmente a los usuarios potenciales si permiten hacer un registro } \\
\text { en video en la entrevista, para posteriores análisis y conclusiones. }\end{array}$ \\
\hline $\begin{array}{l}\text { Ciclo de vida del } \\
\text { proceso }\end{array}$ & $\begin{array}{l}\text { Esta evaluación se realiza en la primera etapa del ciclo de desarrollo del proyecto. } \\
\text { La instalación es una prueba preliminar. }\end{array}$ \\
\hline $\begin{array}{l}\text { Método general } \\
y \text { actividades }\end{array}$ & $\begin{array}{l}\text { El asistente tendrá plena libertad de expresar ideas y comentarios tras el proceso } \\
\text { de interacción. Se instará a que se realicen comentarios que permitan un análisis } \\
\text { profundo de los elementos de la propuesta con base en la instalación. Mientras } \\
\text { los asistentes visiten la instalación, se tomará nota de comentarios y actitudes } \\
\text { frente a cada elemento. }\end{array}$ \\
\hline Plan & $\begin{array}{l}\text { La actividad tomará un tiempo de veinte a veinticinco minutos, en lo que se } \\
\text { incluyen preguntas o comentarios de los asistentes. }\end{array}$ \\
\hline $\begin{array}{l}\text { Personal } \\
\text { responsable }\end{array}$ & $\begin{array}{l}\text { Quien implementa la instalación (profesor Antonio Dorado, en el marco del pro- } \\
\text { yecto Retrato hablado de Augusto Rivera), diseñadores y desarrolladores. }\end{array}$ \\
\hline
\end{tabular}

Fuente: elaboración propia.

\section{Análisis general de la aplicación de la evaluación}

La presentación de la instalación Uno se muere y sigue aprendiendo a pintar fue realizada el jueves 17 de diciembre de 2015, a las 12:00 del día. Acudieron diversas personas, en su mayor parte estudiantes, empleados y profesores de la Facultad de Artes Integradas; ingresaron cuarenta y seis participantes para hacer la evaluación.

El análisis de los registros del video, las observaciones directas y la prueba mostraron que el primer objeto artístico cumplió con su función introductoria (Her, 2014). Esto quiere decir que iniciaron a los participantes en la presentación de un 
artista que desconocían. Se encontró que la mayoría de los asistentes no solo habían escuchado la voz del artista, sino que habían leído, al menos, parte de los textos biográficos porque la mayoría de las personas se habían detenido en ese lugar.

En la segunda obra, Senderos de Rivera, se presentaron variantes importantes. Los registros de cámara evidenciaban que las pinceladas, al proyectarse sobre capas gruesas de humo, se magnificaban. Los entrevistados se refirieron a esta experiencia como una obra que "expresaba la mimesis del gesto de quien pinta sobre el humo" (comunicación personal, 17 de diciembre de 2015). Otros referían "la creación al azar figuras tridimensionales y fantasmagóricas que se proyectaba en diversos colores" (comunicación personal, 17 de diciembre de 2015). En este sentido, el diseño de la obra que se esperaba proyectar de manera bidimensional se transformó en la ejecución, y generó entre los participantes mayor trascendencia de la que se había planificado. Esta situación invitó a la reflexión sobre la manera en que las atmósferas museísticas interactivas crean condiciones de espacios de sentido (Zunzunegui, 2003), donde el contexto involucra y transforma al espectador en participante.

En la tercera obra, denominada Alumbramientos del artista, el empleo sonoro del réquiem de Verdi resignificó los espacios y le otorgó a la pieza y a toda la instalación un sentimiento lúgubre. Los registros de la cámara infrarroja evidenciaron la breve metamorfosis que motivó a otros espectadores a transgredir el territorio de la obra para integrarse en la lúdica de hacer selfis acostados sobre el lienzo que cubría la cama. Esta situación reflejó las transformaciones del rol del espectador que se apropia de la obra y se integra, a través de las nuevas tecnologías, en el espacio museístico como un medio de comunicación (Carmona, 2011).

Finalmente, se encontraba La cena del artista. La evaluación de los asistentes fue positiva, aun en los casos en que el dispositivo tecnológico falló. Esto se corroboró en las entrevistas y en los registros de la evaluación.

En las grabaciones audiovisuales se observó que los dispositivos hápticos que activaban el mapping de obras de Rivera sobre el lienzo en la mesa, propiciaba gestos de asombro y marcaba registros emocionales positivos. El proceso que se gestó alrededor de la obra fue importante como experiencia para los estudiantes y docentes involucrados en el aprendizaje.

La instalación museística reafirma su lugar de interacción social, donde se replica el efecto de los saberes compartidos. La metáfora del comedor hace una remembranza de la época en que la familia se sentaba a conversar alrededor de la comida. En las entrevistas realizadas, esta obra fue subrayada con gran entusiasmo por la participación que convocaba. Al final de la exhibición, las personas subieron 
por las gradas de evacuación del estudio hasta un descanso. Este lugar permitía tener una visión panorámica de todas las piezas de la instalación. Hubo asistentes que aprovecharon el ángulo para tomarse selfis.

De esta manera, se abordaron quince entrevistados que ayudaron a configurar y complementar la evaluación. En las respuestas, se advirtió que ninguno supo responder el nombre de la instalación y pocos recordaron el nombre de Augusto Rivera, el artista indigenista sobre el cual se había realizado la muestra. Les atrajo la idea de la interactividad y manifestaron que les gustaría haber tenido más experiencias similares. Solo un asistente conocía al pintor, los restantes mostraron interés en conocer su obra. Un aspecto generalizado es que, como los visitantes no conocían el repertorio de las propuestas diseñadas, no advirtieron que algo faltara y quedaron complacidos con lo que vieron.

Por otro lado, sobre el recorrido de los asistentes entre las obras, se pudo observar mediante las cámaras infrarrojas que no siguieron el planteamiento sugerido de acuerdo con la disposición de las obras en la instalación. De esta manera, las instalaciones interactivas están transformando los modos de apropiación al permitir que los usuarios se involucren de manera diferente. En este sentido, se fomentan propuestas abiertas donde se estimula el placer de descubrir, pero lo más importante es que son aprendizajes que estimulan los aprendizajes sociales (Alonso, 2015).

Como conclusión general, se puede afirmar que las piezas interactivas son vulnerables y pueden presentar dificultades técnicas. Es conveniente hacer ensayos previos, pues los trabajos interactivos dependen de procedimientos complejos de programación de sensores. Los computadores realizan operaciones, pero sus flujos dependen de condiciones diversas. Además, se tienen diversos tipos de usuarios. En la prueba realizada se observó cómo, en un público cualificado, la mayoría de los asistentes son cuidadosos, pero no faltan los espectadores inquietos quienes, al tener licencia para interactuar, tocan las piezas. En nuestro caso, movieron los platos en la mesa y las servilletas de tela de la utilería; igualmente dañaron algunos audífonos. La situación resultó mucho más compleja de lo que imaginamos con algunos usuarios. Pero otro de los aprendizajes es que las obras interactivas deben estar preparadas para ser intervenidas.

\section{Arquitectura del Algoritmo UX-Brain}

A continuación, en la figura 2, se muestra un esquema de la arquitectura del algoritmo de Ux-Brain en la cual se presentan de manera genérica los elementos que lo conforman (debe aclararse que, en el gráfico, la referencia cliente corresponde al rol del espectador en el contexto del presente trabajo). 
El capturador de datos BCI (EEG) (Ferreira et al., 2019; Al-Nafjan et al., 2017; Folgieri, 2017; Cuartas, Madrigal y Torres, 2011) inicia un agente software que establece la conexión con el controlador de Neurosky ${ }^{\circledR}$. Posterior a ello, cuando se esté ejecutando captura los datos (eSense Data), que está dando el BCI y crea el archivo cuando se destruya o termine, guarda en el sistema de archivos el archivo (*.Csv).

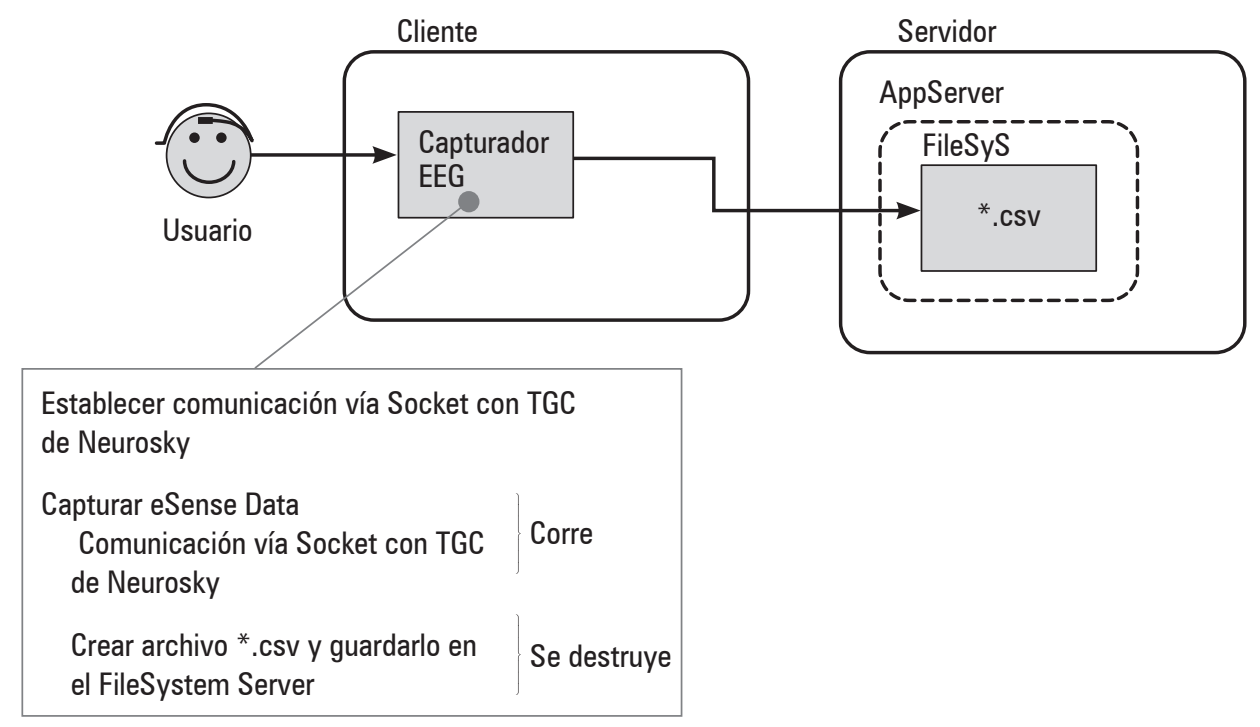

Figura 2. Arquitectura del algoritmo de Ux-Brain

Fuente: elaboración propia.

Finalmente, hace un proceso sobre ese CSV que consiste en calcular la distribución de esos datos en términos del promedio de la atención (A) y la meditación (M), y los escribe en el archivo que quedan guardados en el servidor. Cabe resaltar que toda esta comunicación se hace vía bluetooth (Reyes, 2017).

\section{Aplicación del método UX-Brain}

Las pruebas efectuadas permitieron observar la relación de la meditación y atención de los espectadores en la realización del recorrido dentro de la instalación artística a partir de un camino guiado de observación de los distintos elementos de la obra establecidos en la investigación (Cardona, 2016; Isomursu, 2008). Además, fueron seleccionados aleatoriamente algunos espectadores que usaron el dispositivo Emotiv-epoc ${ }^{\circledast}$, el cual se comunicaba con bluetooth con un computador ubicado estratégicamente detrás de la exposición. 
Por su parte, el facilitador de la prueba monitoreaba los tiempos de la actividad. En el mismo momento en que las actividades se iban haciendo, el dispositivo iba capturando la información necesaria que se iba recopilando en una base de datos de un computador (tabla 2).

Tabla 2. Recopilación de datos de los estados cerebrales de los usuarios.

\begin{tabular}{|c|c|c|c|c|c|c|c|c|c|c|}
\hline Atención & Meditación & Delta & Theta & Low alpha & High Alpha & Low Beta & High Beta & \multicolumn{2}{|c|}{ Low Gama Hight Gama } & Aho \\
\hline & \multirow{4}{*}{34} & 613.704 & \multirow{4}{*}{$\begin{array}{c}485.934 \\
51.367 \\
33.076\end{array}$} & 43.236 & \multirow{4}{*}{$\begin{array}{l}36.855 \\
17.667\end{array}$} & \multirow{4}{*}{$\begin{array}{c}34.652 \\
8.432\end{array}$} & \multirow{4}{*}{$\begin{array}{l}11.917 \\
11.520\end{array}$} & & \multirow[b]{2}{*}{7.013} & \\
\hline & & 24.629 & & 6.926 & & & & 6.816 & & \\
\hline & & 43.927 & & 17.067 & & & & 3.326 & 1.246 & \\
\hline & & 31.638 & & 3.061 & & & & 6.967 & 4.167 & \\
\hline & \multirow{2}{*}{67} & 1.655171 & \multirow{2}{*}{29.824} & \multirow{2}{*}{31.853} & \multirow{2}{*}{21.161} & & 14.760 & 4.922 & & \\
\hline & & 76.589 & & & & & 4.191 & 4.177 & & \\
\hline & \multirow[t]{2}{*}{43} & 343.155 & 147.195 & & 39.061 & 10.244 & 16.750 & & & \\
\hline & & 74.954 & 41.273 & 4.736 & 7.758 & 11.855 & 9.351 & & 21.295 & \\
\hline & 38 & 145.633 & 17.161 & 10.042 & 7.551 & & & & 2.342 & \\
\hline & \multirow{3}{*}{51} & 333.561 & 170.261 & \multirow{3}{*}{40.691} & 31.453 & & \multirow{3}{*}{$\begin{array}{c}23.547 \\
3.379\end{array}$} & \multirow{3}{*}{8.049} & \multirow{3}{*}{$\begin{array}{l}9.452 \\
4.819\end{array}$} & \\
\hline & & 153.894 & 27.066 & & 5.149 & & & & & \\
\hline & & 68.324 & 61.858 & & 11.918 & & & & & \\
\hline & 67 & 196.065 & 19.773 & & & 5.352 & 8.681 & 8.051 & & \\
\hline & \multirow{3}{*}{$\begin{array}{l}80 \\
70\end{array}$} & 21.436 & \multirow{3}{*}{71.676} & \multirow{3}{*}{$\begin{array}{c}6.412 \\
24.153\end{array}$} & \multirow{3}{*}{$\begin{array}{c}8.992 \\
44.168\end{array}$} & \multirow{3}{*}{$\begin{array}{c}4443 \\
14.922\end{array}$} & 20.742 & \multirow{3}{*}{6.572} & & \\
\hline & & 237.647 & & & & & 11.934 & & & \\
\hline & & 349.995 & & & & & 53.145 & & & \\
\hline & \multirow{2}{*}{75} & 308.889 & 68.837 & 71.581 & 61.432 & \multirow{2}{*}{2.255} & 4494 & 1.435 & 4497 & \\
\hline & & 115.146 & 12.164 & 17.436 & 11.783 & & 11.643 & 5.116 & 15.730 & \\
\hline & \multirow{3}{*}{60} & & 46.072 & \multirow{3}{*}{$\begin{array}{l}18.226 \\
15.815\end{array}$} & & & 8.728 & 11.432 & & \\
\hline & & 081.102 & 13.692 & & 10.653 & 14.385 & 6.601 & 3.272 & 3.954 & \\
\hline & & 18.691 & 24.864 & & & 4.913 & 5.979 & 6.595 & 2.490 & \\
\hline & 80 & 131.123 & 47.996 & 21.442 & 18.735 & 6.441 & 14.313 & 12.193 & & \\
\hline & 83 & 294.207 & 7.626 & 8.319 & & 4.273 & 21.992 & & 4.164 & \\
\hline & 69 & 142428 & 10.768 & 3.411 & 1897 & 1.550 & 2.071 & & 1321 & \\
\hline & (2) & & 57.604 & 12.927 & $1.09 \pi$ & 18.076 & 10.851 & & 1.321 & \\
\hline & & & 43.556 & & & & 7.558 & & & \\
\hline & & 38.037 & 25.929 & 24.803 & & 6.579 & 11.061 & 16.493 & 3.272 & \\
\hline & & 22.769 & 11.657 & 20.033 & 9.149 & 14.010 & 9.110 & 1.518 & 2.001 & \\
\hline & & & 27.569 & 8.073 & & 0.422 & 3.672 & & & \\
\hline & & 21.081 & 42.974 & 20.297 & 21.372 & & 11.301 & 5.734 & & \\
\hline & 69 & 109.324 & 55.746 & 20.279 & & 8.597 & 3.423 & 5.578 & & \\
\hline & & 99.524 & 46.517 & & 23.920 & 10.744 & 7.721 & & 3.152 & \\
\hline
\end{tabular}

Fuente: elaboración propia.

Como se ve en la tabla 2, la aplicación desarrollada dentro del proyecto captura los datos de atención y meditación, así como los valores de los diferentes estados 
que se generan en la actividad cerebral: delta, teta, alfa, beta y gama. Esos valores fueron insumos que se cruzaron con las dificultades que se advirtieron a través de la reflexividad de la observación participante. De este modo, se logró corroborar la validez de su aplicación en escenarios de interés artístico. En consecuencia, estos resultados afianzaron los análisis e inferencias que se propusieron para mejorar la interactividad.

\section{Ejemplificación de uno de los momentos particulares de interacción y el resultado de meditación (M) y atención (A) obtenido}

A continuación, la tabla 3 corresponde al momento en el que uno de los usuarios se encontraba interactuando con el primer objeto artístico: introducción.

Tabla 3. Análisis de la A y la M en la franja de interacción

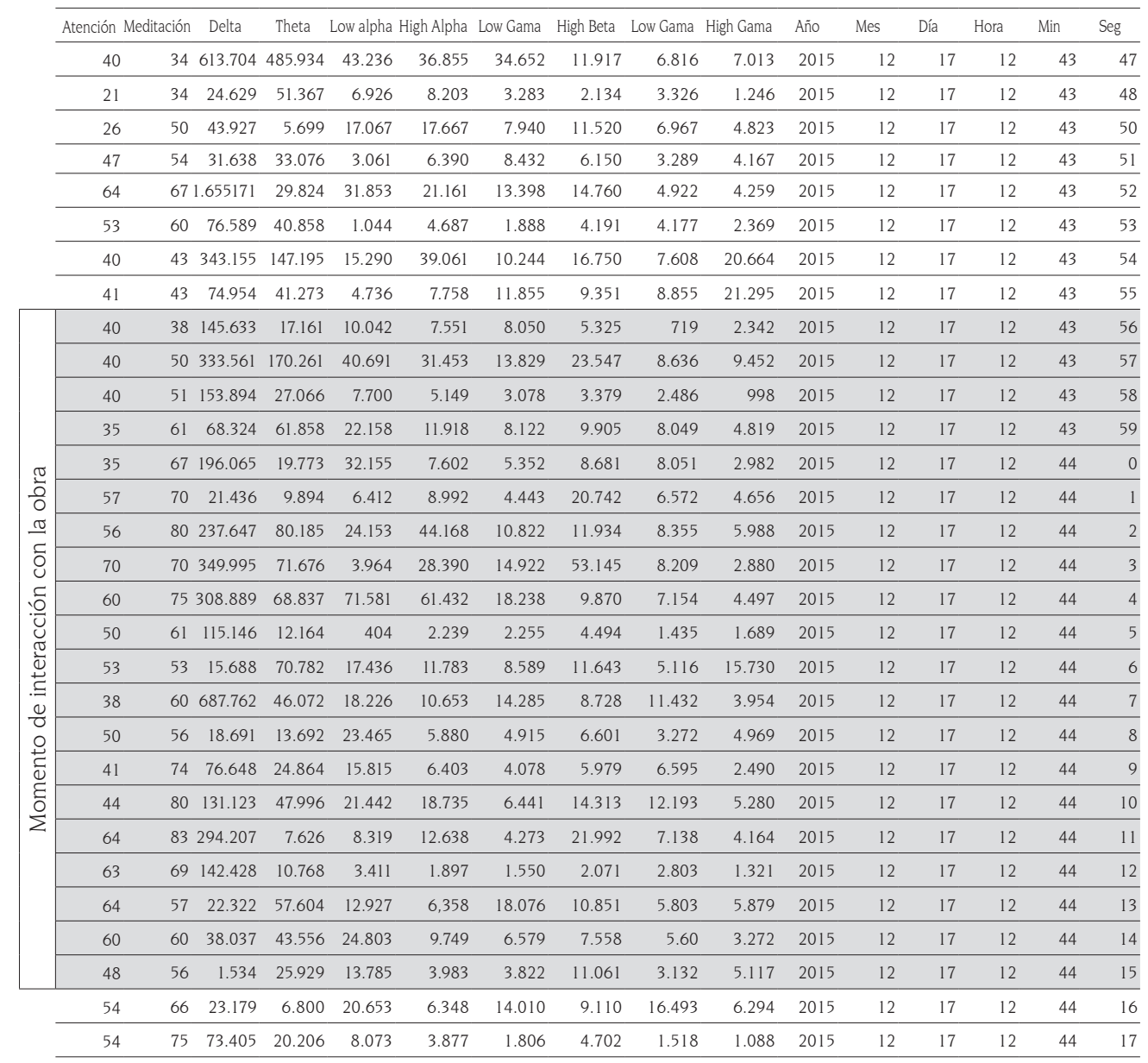




\begin{tabular}{crrrrrrrrrrrrrrrr}
\hline Atención Meditación & Delta & \multicolumn{2}{l}{ Theta } & \multicolumn{2}{l}{ Low alpha High Alpha } & Low Gama & High Beta & Low Gama & High Gama & Año & Mes & \multicolumn{2}{l}{ Día } & \multicolumn{2}{l}{ Hora } & \multicolumn{2}{l}{ Min } & Seg \\
\hline 63 & 70 & 46.694 & 11.657 & 6.868 & 1.004 & 1.806 & 3.672 & 2.020 & 2.001 & 2015 & 12 & 17 & 12 & 44 & 18 \\
\hline 64 & 66 & 22.769 & 27.569 & 6.665 & 7.228 & 6.422 & 13.328 & 3.694 & 3.283 & 2015 & 12 & 17 & 12 & 44 & 19 \\
\hline 57 & 64 & 21.081 & 42.974 & 20.297 & 21.372 & 2.606 & 11.301 & 5.734 & 3.403 & 2015 & 12 & 17 & 12 & 44 & 20 \\
\hline 43 & 69 & 109.324 & 55.746 & 20.279 & 25.884 & 8.597 & 3.423 & 5.578 & 3.396 & 2015 & 12 & 17 & 12 & 44 & 21 \\
\hline 37 & 69 & 99.524 & 46.517 & 7.868 & 23.920 & 10.744 & 7.721 & 4.608 & 3.152 & 2015 & 12 & 17 & 12 & 44 & 22 \\
\hline
\end{tabular}

Fuente: elaboración propia.

De acuerdo con la observación, el usuario estuvo interactuando desde las 12:43:56 hasta las 12:44:15 y se obtuvo un promedio de los estados de atención de 49,5 y de meditación de 63,55. Esto indica que el espectador se encontraba motivado y expectante respecto a la obra, dado que la meditación indica un nivel de confianza. Por otra parte, su nivel de atención (A) era neutral, lo cual se dio por la atmósfera presentada en el escenario.

Cada uno de estos datos obtenidos a nivel cuantitativo por el dispositivo BCI nos permitió orientar las observaciones realizadas en el registro en video para confirmar sus estados de A y de M. Esto nos ayudó a tomar decisiones respecto a lo que debíamos modificar de la propuesta.

\section{Resultado}

Tras compartir la exposición con espectadores reales en el laboratorio, se decidió elaborar una nueva versión. Se afinaron las propuestas de interactividad logradas y se hizo un segundo ensayo en el mismo estudio de televisión — sin público— para ampliar la fase de exploración; se hicieron registros fotográficos y de video pensando en la promoción y en los desarrollos futuros del trabajo. En los nuevos ensayos se afianzó el laboratorio audiovisual de exploración con el humo como un elemento efímero, cambiante, etéreo y fugaz, metáfora de la memoria que permitía proyectar obras de Rivera; se hicieron ensayos con diferentes soportes y obras. A partir de tal experiencia de las proyecciones, se introdujo una pieza capitalizando el azar de las proyecciones sobre el humo y se diseñó la proyección de una animación que Augusto Rivera realizó como afiche para el Festival de Música Religiosa de Popayán. En la animación, un cristo se proyectaba en rayos de luces a través de un back-proyección que se proyecta sobre el humo encerrado en cubo en acrílico. Se reafirmó que las instalaciones son una creación libre e independiente de la obra de Rivera en las que se utilizan sus obras y una investigación biográfica con la que se construyen las diferentes obras objetuales sobre las cuales reposa la propuesta de creación. De alguna manera, se trataba de poner a prueba los resultados del laboratorio y confrontarlo con el gran público de la ciudad. 


\section{Socialización y difusión de resultados}

Una alternativa de dar cuenta de la socialización, la utilidad y el impacto de una obra interactiva y del laboratorio pedagógico se pudo medir por la concertación dialógica en el proceso de aprendizaje entre estudiantes, docentes, pero también en los logros de la obra al participar y ser elegida en franca lid en exigentes escenarios museísticos.

Los indicadores que se obtuvieron transformaron y enriquecieron la propuesta experimental con alternativas de creación y difusión de piezas audiovisuales. La nueva propuesta de instalación interactiva se presentó a consideración del Museo La Tertulia ${ }^{4}$, un espacio con tradición en el ámbito artístico en Colombia y Latinoamérica. La muestra fue aprobada y se exhibió bajo el título Augusto Rivera, el gran ausente, entre el 11 y el 19 de octubre de 2018, en la sala subterránea del Museo. El diseño de la propuesta de medios y la promoción de la exposición circuló en medios locales con impacto en espacios culturales de prestigio. Desde la inauguración, se logró una amplia convocatoria. Para la exhibición se retomaron todos los insumos que arrojaron las evaluaciones del laboratorio. Se elaboró un nuevo texto curatorial con mayor claridad y comprensión sobre la obra y la interactividad que se podía ofrecer. Se revisó el nombre de la obra y se propuso Augusto Rivera, el gran ausente. Exposición inter-activa. El gran ausente hace alusión al retrato que Rivera hace del herrero indígena de su pueblo. Pero el nuevo título, más breve, también involucra el nombre completo del artista porque el apellido puede aludir al muralista mexicano, Diego Rivera. Además, el nuevo nombre incluye la idea de que la instalación trata sobre un gran personaje hoy ausente. En el texto curatorial se amplía la información y se revela que se trata de un gran artista, ausente al haber sido olvidado por los museos y por la historia. El subtítulo Exposición inter-activa indica que la propuesta involucra la participación del espectador.

Para reorganizar el recorrido de la obra, se exploró la sala subterránea del Museo. Se revisaron los planos y las dimensiones que se requerían para cada pieza de la instalación, teniendo en cuenta la altura necesaria para los proyectores, ubicación de computadores, distancias, orientación del sonido, etc. Fue importante tener el background de la investigación sobre el artista, la evaluación de interactividad realizada y el guion curatorial que sostenía la muestra. Con respecto a las consideraciones sobre la exploración de las pantallas expandidas, la sala subterránea no solo resultó apropiada porque estaba ubicada bajo el nivel del edificio, sino porque en ese espacio se creó una especie de caverna subterránea que facilitó el control de la luz. Se compuso, a partir de esta, una extensión de una sala de cine donde primaban las luces de los proyectores sobre los objetos en un ambiente de humo que trasmitía

http://www.museolatertulia.com/museo/exposiciones/augusto-rivera-gran-ausente/ 
cierto "olor azufrado" que la crítica Martha Traba había advertido en sus obras. El azar se hizo cómplice del homenaje que rendimos a Rivera.

En este mismo espacio se entregó un programa de mano que amplía la comprensión de la obra y describe cada elemento para que el espectador tenga una información más amplia sobre la mirada que pretende compartir la instalación.

Considerando que no todos los espectadores leen todo lo que ven —o lo que se les entrega-, el museo dispuso dos guías (estudiantes de bellas artes) que orientaban, a través de un guion museográfico preestablecido, el vínculo que se pretendía establecer entre el artista homenajeado y el carácter interactivo de las obras. Esta tarea resolvió muchos interrogantes y lagunas que había arrojado la prueba.

Adicionalmente, en la pared central de la antesala del museo se reelaboró y complementó la obra introductoria sobre el artista: comienza con un texto aclaratorio sobre sus fechas de natalicio y muerte; incluye un fragmento de un archivo fílmico de Rivera con un lienzo en blanco al fondo donde se dispone a pintar; a continuación, se proyecta una secuencia de obras originales de Rivera porque en las instalaciones posteriores estarán intervenidas; finalmente, incluye un caligrama que revela poco a poco el rostro del artista hasta crear la imagen de la marca y el nombre de la instalación. Para concluir, los objetos de exposición se dispusieron en la sala a partir de una ruta ideal, pero considerando que cada pieza debía funcionar de manera independiente. Se asumió que, si se rompía ese orden, no afectaría la propuesta.

Para la inauguración y la promoción del evento, se elaboró una estrategia mediática de pequeña escala, similar a la del lanzamiento de una película. Se diseñó un afiche que se colocó en espacios visibles de la ciudad, se produjo un tráiler que se difundió por canales de televisión local y regional, se imprimieron postales que se distribuyeron en universidades y lugares estratégicos para la actividad cultural de la ciudad. Adicionalmente, Gaceta - la revista cultural de mayor circulación en la ciudad - hizo eco de la organización de la instalación y publicó un artículo central, además de que la tomó como elemento de portada. Estas piezas circularon en las principales redes sociales y se alcanzó un impacto inusual para este tipo de eventos. Para la inauguración, se programó un conversatorio del director de la instalación con dos personajes conocedores de Rivera: el arquitecto Álvaro Thomas, amigo personal del artista, y el actor de cine y teatro Humberto Dorado, quien conoció de cerca al artista. Finalmente, se logró el objetivo de difundir la obra de Augusto Rivera a través del homenaje. Además, se hizo un trabajo creativo para el museo apelando a la interactividad y al documental expandido y fusionando estas alternativas como estrategias para convocar a los públicos convencionales y a las nuevas generaciones. 
En los tiempos previstos la obra se abrió al público y funcionó correctamente. Sin embargo, a partir del segundo día surgió de nuevo el problema de la complejidad tecnológica advertida con la prueba: la programación que se concibió para la mesa, después de funcionar a la perfección inicialmente, dejó de funcionar. La pieza Insolencias benignas, que activa al tacto la proyección visual y sonora de una obra de Rivera, funcionó bien casi todo el tiempo, pero no resistió una visita de estudiantes de colegios. Sin embargo, con la experiencia acumulada, las dificultades se lograron subsanar. El balance general fue afortunado.

Posteriormente, una de las piezas de la instalación (Insolencias benignas) fue seleccionada en el Festival Internacional de la Imagen 20195. Adicionalmente, el laboratorio y la prueba han servido como insumo para la tesis doctoral ${ }^{6}$ que adelanta el artista de la obra en la Universidad de Granada, y ha sido de gran utilidad para simular estados y problemas potenciales, lo que configura un recurso importante para orientar a estudiantes y creadores frente a la incertidumbre sobre la funcionalidad de sus obras. Estas pruebas son esenciales para los procesos creativos y muestran aspectos que los creadores no advierten en la concepción de las obras.

\section{Discusión y conclusiones}

La estrategia de crear un laboratorio de instalaciones interactivas en condiciones controlables, extrapolando los laboratorios vanguardistas en el contexto poiético de una producción artística interactiva que se retroalimenta con la experiencia entre estudiantes, docentes y público, se mostró válida. La implementación de la observación participante, a través de la estrategia de camuflar a los estudiantes entre la oscuridad y el público, fue valiosa. Esta situación no solo facilitó la ejecución de los registros videográficos y fotográficos, sino que permitió reforzar la evaluación de la interactividad en la oscuridad. Los datos recopilados del BCI permiten comprender el comportamiento y las relaciones cronométricas, tímicas y proxémicas entre los espectadores y las obras artísticas. Este proceso permitió, a la postre, enfrentar las dificultades pragmáticas para asumir los correctivos respectivos. La extrapolación del planteamiento de Bruno Latour, es decir, sacar la propuesta del laboratorio y confrontarla en su contexto social, no solo se muestra válida para afianzar su implementación comunicacional, sino como alternativa pedagógica de un aprendizaje colectivo horizontal.

http://festivaldelaimagen.com/programacion/

6 La tesis se titula Los misaks, de la tierra a la pantalla. Creación audiovisual en el resguardo indígena de Wuambia. La investigación aborda la creación de una metodología audiovisual que indaga alternativas de producción audiovisual y recursos alternativos de pantallas expandidas como piezas para museo. 
De igual manera, se confirma que sí es posible aplicar una prueba para orientar un sistema de evaluación que permita identificar, comprender y retroalimentar de manera creativa las ideas sobre problemas y virtudes en procesos de interactividad en piezas artísticas para museo. Evidentemente, los métodos de indagación empleados en el proceso de la instalación son altamente positivos. La metodología empleada por el profesor Javier Reyes en la investigación Emot-brain: método de evaluación de experiencia de usuario no solo es funcional para evaluar experiencias de usuarios en computadores, sino que se puede aplicar con usuarios de piezas tridimensionales interactivas.

La prueba es aplicable y permite detectar dificultades y alternativas de soluciones. Adicionalmente, mediante las entrevistas y los registros visuales se pueden recibir evaluaciones complementarias que fortalecen los procesos de interactividad puestos al servicio del trabajo creativo. Las evaluaciones de la prueba son pistas que permiten identificar virtudes, problemas potenciales o tendencias positivas o negativas. Como se trata de proyectos artísticos expuestos a reacciones subjetivas y responden a actos o reacciones fisiológicas o mecánicas, no necesariamente son de aplicación directa. En consecuencia, no deben tener un carácter impositivo. Las pruebas recogen apreciaciones de un equipo de trabajo que se plantean como recomendaciones, y estas pueden ser acogidas parcial o totalmente por el artista o el colectivo que ha diseñado la propuesta.

El método de la prueba arroja un diagnóstico confiable y, a su vez, indica una ruta que pone en contacto al creador artístico con su público potencial. Esta situación puede arrojar resultados contundentes de retroalimentación que acercan al usuario con el artista para fortalecer sus vínculos. Esta expansión de la aplicación de la prueba al ámbito artístico funciona como alternativa para encontrar y explorar otros escenarios de proyección y de reencuentro con el público. Sin embargo, el desafío de la producción artística contemporánea es conservar una distancia crítica en la saturación del empleo de tecnologías interactivas. En la búsqueda de apuestas novedosas de destrezas hápticas hay que tener mesura, e inclusive considerar alternativas de producción artística que permitan crear obras que involucren a los espectadores en la construcción de un sentido crítico capaz de subvertir y cuestionar la amenaza de apropiación gratuita de tecnologías (Muollo, 2019).

La propuesta del laboratorio de interactividad y la aplicación de la prueba constituyen una importante herramienta pedagógica que se puede replicar en otros proyectos de complejidad similar para afianzar, a través de la interdisciplinariedad, estrategias de cualificación en la investigación artística. 
Cuando los espectadores, se involucran como participantes y se apropian colectivamente de saberes, el impacto emocional es mayor. Esta es una ruta importante para interpretar la interactividad como una alternativa emancipatoria. Es una apuesta que no responde simplemente a un condicionamiento reflejo, sino que convoca a un escenario de descubrimientos y aprendizajes lúdicos que se reafirman socialmente.

\section{Referencias}

Al-Nafjan, A., Hosny, M., Al-Ohali, Y. y Al-Wabil, A. (2017). Review and classification of emotion recognition based on EEG brain-computer interface system research: A systematic review. Applied Sciences, 7(12). https://doi.org/10.3390/app7121239

Alonso, R. (2015). Introducción a las Instalaciones Interactivas. Centro de Estudios En Diseño y Comunicación, 51, 51-64.

Bordwell, D. (1999). El cine de Eisenstein: teoría y práctica. Paidos.

Cardona, F. T. (2016). Catálogo del XV Festival Internacional de la Imagen del Diseño y la Creación para el biofeedback. Manizales, Balance Unbalance.

Carmona, R. M. (2011). Discurso audiovisual y cultura digital: comunicación e interactividad en los museos. En C. Mateos Martin (Coordinador)En C. Mateos, A. Ardévol y S. Toledano (eds.), III Congreso Internacional Latina de Comunicación Social, España. http://www.revistalatinacs. org/11SLCS/actas_2011_IIICILCS/126_Marfil.pdf

Centro Virtual Cervantes (2008). Lengua. Consultado el 8 Junio 2020. https://cvc.cervantes.es/ lengua/default.htm.

Cuartas, J. A., Madrigal, P. A. y Torres, R. A. (2011). Interfaz por sincronización y desincronización relacionada a eventos en sujetos no entrenados. En F. Méndez, T. Y. Aznielle, C. Calderón, S. Llanusa, J. Castro, H. Vega, M. Carballo, R. Rodríguez (eds.), V Latin American Congress on Biomedical Engineering CLAIB (pp. 611-614) IFMBE Proceedings. https://doi. org/10.1007/978-3-642-21198-0_156

Dorado, A. (2016). Augusto Rivera, memorias del olvido. Revista Nexus Comunicación, (19), 60-83. https://doi.org/10.25100/nc.v0i19.664

Ferreira, C. A., Cunha, A., Mendonça, A. M. y Campilho, A. (2019). Convolutional Neural Network Architectures for Texture Classification of Pulmonary Nodules. En R. Vera-Rodriguez, J. Fierrez y A. Morales (eds), Progress in Pattern Recognition, Image Analysis, Computer Vision, and Applications. CIARP 2018. Lecture Notes in Computer Science, vol 11401. Springer, Cham. https:// doi.org/10.1007/978-3-030-13469-3_91

Folgieri, R. (2017). Brain Computer Interface and Transcranical Stimulation: Frontiers, reliability, safety and threats. BioLaw Journal, (3), 103-110. https://doi.org/10.15168/blj.v0i3.262

Guber, R. (2001). La Etnografia: método, campo y reflexividad. Editorial Norma.

Heimonen, T., Aula, A. y Hutchinson, H. (2008). Comparing the user experience of search user interface designs. Position paper to be presented in the workshop "Now let's do it in practice: user experience evaluation methods in product development" at CHI' 2008. 
Her, J. J. (2014). An analytical framework for facilitating interactivity between participants and interactive artwork: case studies in MRT stations. Digital Creativity, 25(2), 113-125. https:// doi.org/10.1080/14626268.2013.776974

Isomursu, M. (2008). User experience evaluation with experimental pilots. CHI.

Knapp, L. (2009). La comunicación no verbal. El cuerpo y el entorno. Editorial Paidós Mexicana.

Latour, B. (1983). Dadme un laboratorio y levantaré el mundo. Organización de Estudios Iberoamericanos para la Ciencia y la Cultura.

Lin, T. (2013). Attraction and action. User types blending aesthetical and functional design. IEEE.

Mahrin, M. S. (2009). Selecting Usability Evaluation Methods for Software Process Descriptions. 16th Asia-Pacific Software Engineering Conference, Penang. https://ieeexplore.ieee.org/ document/5358871

Madan, A. y Kumar, D. S. (2012). Usability evaluation methods: a literature review. International Journal of Engineering Science and Technology. 4(2), 590-599. https://www.researchgate.net/ publication/266874640_Usability_evaluation_methods_a_literature_review/fulltext/5444c 1290cf2a6a049ablf0f/Usability-evaluation-methods-a-literature-review.pdf

Manovich, L. (2005). El lenguaje de los nuevos medios de comunicación: la imagen en la nueva era digital. Ediciones Paidós.

Mariniello, S. (1992). El cine y el fin del arte. Teoría y práctica cinematográfica en Lev Kuleshov. Cátedra Editorial.

Muollo, G. (2019, 7 de mayo). El objeto como interfaz en una instalación performática interactiva. VI simposio internacional de innovación en medios interactivos. https://files.cercomp.ufg.br/weby/ up/777/o/15_El_objeto_como_interfaz_en_una_instalacio\%CC\%81n_performa\%CC\%81tica_ interactiva_.pdf

Navarro-Newball, A., Moreno-Sanchez, I., Arya, A., Prakash, C., Mike-Ifeta, E. y Mejia-Mena, J. (2017). An Interactive Modelling Architecture for Education And Entertainment at Museums. DYNA, 92(3). 269-273. http://dx.doi.org/10.6036/8058

Navarro, J., Hebner,S., Yenisetti, S., Bayersdorfer, F., Zhang, L., Voigt, A., Schneuwly, S. y Botella, J. (2014). Analysis of dopaminergic neuronal dysfunction in genetic and toxin-induced models of Parkinson's disease in Drosophila. In Journal of neurochemistry, 131, 369-382.

Penny, S. (2011). FCJ-132 Towards a performative aesthetics of interactivity. The Fibreculture Journal, (19), 72-109. http://fibreculturejournal.org/wp-content/pdfs/FCJ-132Simon\%20Penny.pdf

Restrepo, E. (2016). Nómadas etnografía: alcances, técnicas y éticas. Primera Ed.

Rodríguez, P. (2013). Método automatizado para evaluación de usabilidad en sistemas e-learning usando monitoreo de actividad cerebral [tesis de doctorado]. Repositorio Universidad Nacional de Colombia.

Reyes, J. (2017). YUTO Modelo de adaptatividad de ayudas para entornos e-learning Itesis de maestría]. Repositorio Universidad del Valle. 
Sedeño, A. M. (2015). Prácticas de activismo audiovisual con objetivo de integración social: el caso del colectivo Cine sin Autor (CsA). Revista Latinoamericana de Comunicación, (129), 181-192.

Velásquez, P. (2012). Evaluación de la usabilidad de herramientas manuales cotidianas [tesis de pregrado]. Universidad del Valle.

Yonglei, T. (2005, 22 de octubre). Work in progress - introducing usability concepts in early phases of software development. Proceedings Frontiers in Education 35th Annual Conference, Indianopolis, IN. https://ieeexplore.ieee.org/document/1611963

Zunzunegui, S. (2003). Metamorfosis de la mirada. Museo y semiótica. Cátedra Editorial y Universitat de València. 